\title{
Symptoms and Risk Factors of Different Types of PTSD
}

\author{
Ruoxi Liu ${ }^{1}$ \\ ${ }^{1}$ Chengdu No.7 high school, Chengdu, Sichuan, 610041, China \\ Corresponding author's e-mail: ShiLiShuang@cas-harbour.org
}

\begin{abstract}
This paper presents psychological symptom and examples of the patient with PTSD. Different types of PTSD correspond to different risk factors including gender, age, social support and etc. This article presents four common types of PTSD and their symptoms and analyzes overlapping risk factors of examples. It aims for discovering relationship between the risk factors and risk of developing PTSD. It is determined why certain group of people are more likely to develop PTSD considering gender and age.
\end{abstract}

Keywords: Post-traumatic stress disorder, symptom, gender difference, earthquake, amputee, risk factor

\section{INTRODUCTION}

Post-traumatic disorder is a mental illness that is triggered by horrible events such as the death of family members or intimate friends, beings diagnosed with a serious illness, experiencing death threats or serious damages to their physical health, etc. Its symptoms usually include severe depression, general anxiety disorder, addiction, etc. The symptoms of PTSD may be delayed for several years because the traumatic memories are deposited in the procedural memory. When patients perform a certain action, it will trigger the symptom [1]. Nowadays, it is still unclear why only some people who experience traumatic events are diagnosed with PTSD. Research indicates that PTSD is heritable, and the risk of PTSD mutations occurring from inherited PTSD is around $5 \%$ to $20 \%$. PTSD is associated with lower academic achievement, and increasing incidence of depression, suicide attempts, and substance abuse into adulthood [2]. This essay is focusing on the causes of PTSD on soldiers. Researchers nowadays have developed animal model of PTSD such as forcing swimming model, predator exposure model, which is useful for exploring causes, mechanism, and medical treatment of PTSD. It can constantly follow the condition throughout the course of disease in different times and locations, and directly collect samples from ventricles and brain tissues to detect changes in the dynamics of certain biochemical substances and abnormal changes in neurophysiology. Moreover, it can examine the effect of therapeutic drugs and explore the application of new drugs and study the relationship between symptomatology and abnormal changes of some biochemical substances. However, it is still lack of research of its mechanism of its happening, development and commonly used treatment, and its animal model.

\section{DIFFERENT CAUSES OF PTSD}

\subsection{War}

It is known that many American solider suffer PTSD after World War II, but this phenomenon did not occur during World War I. The basic reason is due to the unjust nature of war. In World War II, enormous unjust war take place such as Vietnam War, Afghanistan War, Bay Ear etc. The key influence is the Iraq War, even the woman and kids wanted to kill them. Solider suffered death and massacre every day and killed thousands of ordinaries such as woman or kids, in which led to the self-accusation and guilty in their mind. Meanwhile, to see the intimate comrade-in-arms die in front of them, solider would suffer huge mental pressure. For woman in the troop, they suffer rape and bully without any true actions performed by the government. Unfortunately, after the war, American governments did not consider this important, they only pay compensation for them without any mental treatment. At the same time, they also did not have stable source of income, so they cannot support their own lives or have any social status in the society. Naturally, the negative mood emerged, most of them become and anxious and violent, even some of them committed 
suicide. Therefore, it is highly likely for soldiers to develop PTSD [3].

\subsection{Earthquake}

To test the possible risk factors for PTSD, the selfcompiled trauma exposure screening questionnaire with 11 items was designed to understand the traumatic exposure of the subjects in the Wenchuan Earthquake in 2008. Each item required the subjects to make a choice according to their own experience. The prevalence of PTSD among survivors was $13.8 \%$ after five and a half years, which were similar to those of studies conducted shortly after the Wenchuan earthquake. It suggested that PTSD might be a delayed and persistent mental health problem among earthquake survivors, and patterns of exposure to trauma may differ at different stages [4]. The risk factors include the older age, witnesses of mutilated bodies, death of family members, exposure to trauma gender, and education [4].

\subsubsection{Exposure to trauma and age}

In terms of the exposure to trauma, several previous studies have reported a high correlation between traumatic exposure, such as witnessing the death in an earthquake, and PTSD. However, these traumatic exposures were not found to be a risk factor for PTSD in some studies. This may due to the different tested time about Wenchuan earthquake, suggesting PTSD, as a delayed and persistent disorder, may have different patterns of exposure to trauma at different stages [4]. As for the age, its correlation with PTSD was unclear.

\subsubsection{Gender}

As for the gender, $11.7 \%$ of males are likely to develop PTSD, while $13.8 \%$ of females are more likely than men to develop PTSD after experiencing trauma, while it also suggests that different types of traumatic events may play a role in this gender-based difference. There are four typical types of PTSD: B) invasion symptoms, (C) active avoidance symptoms, (D) negative emotional and change in cognition symptoms and (E) high arousal state symptoms [4].

For the treatment, we need to pay sustained attention to the mental health of survivors of natural disasters. The government should provide more ongoing services and funding for the affected population, and individuals should conduct long-term effective interventions for PTSD. Moreover, Higher educational level is a possible protective factor for PTSD. Survivors of education level of ascension may reduce the risk of PTSD. Focusing resources on timely and effective interventions for these high-risk groups will help reduce the overall incidence of POST-disaster PTSD.

\subsubsection{Parents who have lost their only children}

The possible risk factors may include gender, age, number of years after the death of students, mental resilience, and religious belief. There are five potential categories of PTSD: High PTSD symptom group, moderate PTSD symptom group, low PTSD symptom group and resilience group [5].

\section{GROUP BY SEVERITY}

\subsection{High PTSD symptoms group}

The symptoms include higher psychological and physical manifestations such as flashbacks, loss of interest, emotional numbness, hypervigilance, panic, having difficulty of concentrating and falling asleep [5].

\subsection{Moderate PTSD symptom group}

The symptoms include recalling memories associated with the event repeatedly, psychological, behavioral and physical manifestations of pain, avoidance of relevant places, events and people, and difficulty of falling asleep [5].

\subsection{Low PTSD symptom group}

The symptoms include psychological, behavioral, and physical manifestations of internal pain, avoidance of relevant memories, people and places, and difficulty falling asleep [5].

\section{RESILIENCE GROUP}

To sum up, there are some differences in the manifestations of core symptoms of PTSD among different categories, suggesting that there is population heterogeneity in PTSD symptoms of orphaned-parents. This indicates characteristics of complexity and diversity of PTSD.

In terms of mental resilience, generally, it refers to the individual's ability to recover from negative experiences. The ability of higher mental toughness helps the individual bear setbacks, promoting individuals to recover from trauma experience. Previous studies have also found that psychological resilience can effectively alleviate the adverse adaptive results after the death of a single child. The lower the level of resilience, the higher the likelihood of developing PTSD symptoms, that is, the psychological resilience plays an important protective role in the PTSD symptoms of parents who have lost their only child.

As for the gender, the proportion of women with symptoms was higher than males. This suggests that the symptoms of PTSD are more pronounced in the mothers who lost their only child. The possible explanation is that 
mothers, as primary caregivers for their children, devote more time and energy to their children and have stronger emotional bonds with their children than fathers do. Another possibility is that there is a gender difference in how parents cope after losing a child. Mothers are more likely to be emotionally focused, while fathers more likely to be problem-focused.

In terms of age, the younger the parents were, the higher the level of PTSD symptoms. A possible explanation is that older people are more psychologically prepared for death and loss of social functioning and have a lower stress response. In terms of religious belief, studies have found that religious belief, as a way of individual spiritual sustention, can effectively alleviate the psychological pain of parents who have lost their only child.

In terms of the treatment, relevant organizations or institutions can help parents who have lost their only child improve their mental resilience through public lectures, training and other means, and thus to reduce the traumatic reaction after the lost only child. More attention and intervention should be given to females, younger age groups and individuals with lower levels of mental resilience

\section{THE AMPUTEE}

Lack of social support after traumatic events is one of the strongest predictors of PTSD. Social support can be divided into positive social support and negative social support. Different types of social support will lead to different psychological outcomes of trauma patients [6]. PTSD was associated with the time nodes after amputation [6].

The positive rate of PTSD increased on day 3, day 28, and day 84 after amputation. On the 168th day, there was a significant decrease compared with the previous nodes. The increase in positive rate of PTSD refers to the explanation that most of the patients in this study were the main bearers of family subsistence. Amputation made them lose part of their ability to work, which brought great impact on individuals and families. As the main providers of social support, family members and friends are also under different degrees of psychological pressure and prone to generate negative emotions, thus making patients feel a certain degree of negative social support. The positive rate of PTSD decreased 168 days after amputation. The reasons might be as follows: Accepting the change of self-image over time; Various compensatory functions of the healthy limbs gradually formed, and some self-care ability was restored [6]. The PTSD symptoms and social support status of amputees were constantly changing at different time points. The more negative social support patients had, the less positive social support patients had, and the more likely they were to have PTSD [6].

\section{ANALYSIS}

In terms of the examples of PTSD above, we can see that the overlapping risk factors mainly focus on gender, age, and social support. We should consider gender and age together.

As for age, women and men are found to show different lifespan distributions of PTSD. According to Thompson, Norris, and Hanacek, younger people are less subject to disasters, while middle-aged people are more susceptible to disaster. Some plausible explanations indicate that vulnerability to PTSD could be influenced by exposure to traumatic events during different phases of lives such as the menstrual or reproductive cycles. Besides life phases, potential changes in self-perception, social participation, world beliefs, and adaptation to social gender roles also show impact on vulnerability to PTSD. For males, gradual rise in the risk of PTSD has to do with changes in gender roles, tied with work and family responsibilities, causing potentially more stress. Some studies show that old age is associated with low risk of PTSD due to acceptance of experiences in early life and the death. According to previous research, risk of PTSD is related to ego integrity and despair in old life. If ego integrity, wisdom, and life satisfaction are reached, risk of PTSD could decrease both in males during $50 \mathrm{~s}$ and $60 \mathrm{~s}$ and females during the late $50 \mathrm{~s}, 60 \mathrm{~s}$, and early $70 \mathrm{~s}$ and vice versa. Addition studies show that reaching the age of 80 or more might develop new issues in psychosocial development, which could influence risk of PTSD.

As for gender, different research indicates different results. Some results show that women are more likely to develop PTSD during their lifetime. Women showed a higher level of PTSD prevalence from young age to adulthood than men.

Kessler and his colleagues concluded that males show no obvious variation of PTSD rates during different ages. However, when age increases, PTSD rates for women tend to decrease.

Even some arguments have been made that the increased PTSD prevalence among women is due to a report bias resulted from gender role because men prefer to under-report, while women prefer to over-report symptoms of PTSD

\section{TREATMENT}

These results suggest that medical staff should carry out targeted psychological care to reduce the incidence of PTSD while controlling patients' pain. They should carry out psychological care according to the specific situation of patients, from multiple ways, angles and targeted, encourage and guide family members and friends to give amputees more positive social support, promote their emotional communication, so as to better prevent the 
occurrence of PTSD. Professional support, family support and volunteer support form a comprehensive social support model, which can not only improve the patient's overall quality of life, physical and mental adaptability, but also promote the establishment of a good doctor-patient relationship. It is suggested that the participation of medical staff and volunteers can provide more positive social support for patients.

\section{CONCLUSION}

PTSD will be triggered by different types of traumatic event which related to several risk factors including gender, age, and social support. Different levels of those risk factors will lead to different symptoms and degree of PTSD. As a result, people should take targeted treatment for each type of PTSD triggered by different risk factors. According to the examples, females might more likely to suffer from PTSD than males, and the correlation between age and PTSD depends on internal factors including self-perception or gender roles, and external factors involving stress in daily lives. Moreover, gender and age are tied together to consider the symptoms.

\section{ACKNOWLEDGMENT}

I would like to thank Jasmine, who gave me advices.

\section{REFERENCES}

[1] S. Maren, et al. The contextual brain: implications for fear conditioning, extinction and psychopathology. Nature reviews. Neuroscience, vol. 2013, 14(6): 417-28. Doi:10.1038/nrn3492

[2] R.J. Herringa. Trauma, PTSD, and the Developing Brain. Current psychiatry reports, 19 Aug. 2017, 19(10): 69. Doi:10.1007/s11920-017-0825-3

[3] Sohu. Post-traumatic stress disorder: Why does it become the sole mental illness of the US military? Too many unjust wars. July 23, 2020. Retrieved from:

https://www.sohu.com/a/409279419_120594864

[4] R. Fang, C. Cao, G. Li, etc. Survey of symptoms of post-traumatic stress disorder in survivors five and a half years after the Wenchuan earthquake: based on DSM-5 diagnostic criteria. Psychology and Behavior Research, 2019, 17(01): 109-115.

[5] A. Bouvizorem, H. Zheng, Z. Ma, et al. The potential categories and related factors of post-traumatic stress disorder symptoms in parents who have lost only one. Psychological Science, 2019, 42, 242(06): 227-234.

[6] D. Liu, X. Li, S. Li, Q. Cheng. Longitudinal study of post-traumatic stress disorder and social support in amputees. Chinese Journal of Nursing, 2019, 054(007): 965-969. 